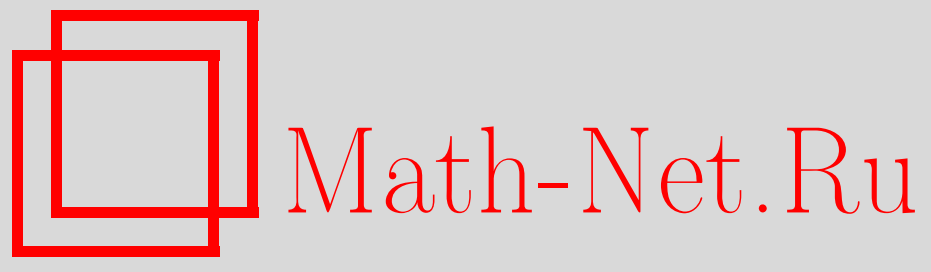

В. Э. Адлер, А. Б. Шабат, Модельное уравнение теории солитонов, ТМФ, 2007, том 153, номер 1, 29-45

DOI: https://doi.org/10.4213/tmf6119

Использование Общероссийского математического портала Math-Net.Ru подразумевает, что вы прочитали и согласны с пользовательским соглашением http://www . mathnet.ru/rus/agreement

Параметры загрузки:

IP: 34.229 .45 .116

26 апреля 2023 г., 14:55:01

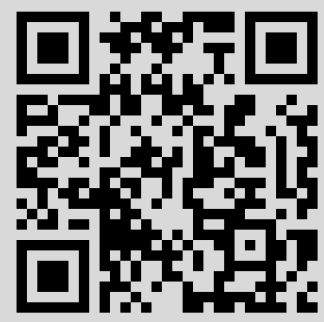




\section{МОДЕЛЬНОЕ УРАВНЕНИЕ ТЕОРИИ СОЛИТОНОВ}

Рассматривается иерархия интегрируемых $(1+2)$-мерных уравнений, связанная с алгеброй Ли векторных полей на прямой. Построены решения в квадратурах, содержащие $n$ произвольных функций одного аргумента. Основной интерес представляет простое уравнение для производящей функции иерархии, определяющее динамику по отрицательным временам и имеющее приложения к спектральным задачам второго порядка. Рассмотрение его полиномиальных решений при условии регулярности соответствующего потенциала позволяет развить довольно общую теорию интегрируемых $(1+1)$-мерных уравнений.

Ключевые слова: иерархия коммутирующих векторных полей, инварианты Римана, уравнения Дубровина.

\section{1. ВВЕДЕНИЕ}

В работе изучается уравнение

$$
D_{\tau} G(\lambda)=\frac{\langle G(\lambda), G(\mu)\rangle}{\lambda-\mu},
$$

где $G(\lambda)=G(x, \tau, \lambda)$ - искомая функция, локально-аналитическая по $\lambda$, и

$$
\langle f, g\rangle \stackrel{\text { def }}{=} f g_{x}-g f_{x} .
$$

Предположение об аналитичности является существенным, так как без него решение содержит слишком большой произвол (ср. ниже, пример 1).

Решение задачи Коши с начальными данными $G_{0}(\lambda)=G_{0}(x, \lambda)$ можно искать в виде ряда Тейлора

$$
G(\lambda)=G_{0}(x, \lambda)+\tau G_{1}(x, \lambda)+\tau^{2} G_{2}(x, \lambda)+\cdots .
$$

Подстановка его в уравнение приводит к рекуррентной формуле

$$
(n+1) G_{n+1}(\lambda)=\frac{1}{\lambda-\mu} \sum_{k=0}^{n}\left\langle G_{k}(\lambda), G_{n-k}(\mu)\right\rangle, \quad n=0,1, \ldots,
$$

${ }^{*}$ Институт теоретической физики им. Л.Д. Ландау РАН, Черноголовка, Московская обл., Россия. E-mail: adler@itp.ac.ru, shabat@itp.ac.ru 
позволяющей вычислить все коэффициенты, если $G_{0}$ - гладкая функция переменных $x$ и $\lambda$. Заметим, что если $G_{0}$ - полином по $\lambda$ степени $m$, то из рекуррентной формулы следует, что и все $G_{k}(\lambda)$ суть полиномы степени не выше $m$. Нетрудно получить оценку для всех коэффициентов ряда и доказать, что он имеет ненулевой радиус сходимости. Таким образом, динамика по $\tau$ сохраняет полиномиальную структуру, что выделяет класс достаточно содержательных решений.

Дифференцирование $D_{\tau}$ зависит, очевидно, от выбора точки $\mu$ в уравнении (1.1). Оказывается, что этот произвол приводит к совместным друг с другом динамическим уравнениям. Точнее, имеем следующее утверждение.

Теорема 1. Пусть дифберениирование $D_{i}$ определяется формулой (1.1) с $\mu=$ $\mu_{i}$. Тогда $\left[D_{1}, D_{2}\right]=0$.

ДокАЗАтЕЛЬСтво. Обозначим $g_{i}=G\left(\mu_{i}\right)$. Из уравнения (1.1) следует, что

$$
D_{1} g_{2}=\frac{\left\langle g_{2}, g_{1}\right\rangle}{\mu_{2}-\mu_{1}}=\frac{\left\langle g_{1}, g_{2}\right\rangle}{\mu_{1}-\mu_{2}}=D_{2} g_{1} \text {. }
$$

Легко проверить, что тождество Якоби

$$
\left\langle\left\langle G, g_{1}\right\rangle, g_{2}\right\rangle+\left\langle\left\langle g_{2}, G\right\rangle, g_{1}\right\rangle+\left\langle\left\langle g_{1}, g_{2}\right\rangle, G\right\rangle=0
$$

обеспечивает равенство $\left[D_{1}, D_{2}\right](G)=0$. Теорема доказана.

Очевидно, что теорема 1 остается справедливой, если заменить $\langle$,$\rangle на скобку$ в произвольной алгебре Ли. Исходная скобка отвечает алгебре Ли векторных полей на прямой; другим интересным примером является скобка $\langle f, g\rangle=f_{y} g_{x}-f_{x} g_{y}$, отвечающая гамильтоновым векторным полям на плоскости. Мы ограничиваемся простейшим случаем, так как и он уже вполне нетривиален и хорошо демонстрирует возникающие здесь задачи.

Приведенное выше уравнение для функций $g_{1}, g_{2}$ можно записать в виде уравнения с частными производными $\partial_{\tau_{i}}=D_{i}$ для потенциала $u$, определенного равенствами $g_{i}=u_{\tau_{i}}$ :

$$
\left(\mu_{i}-\mu_{j}\right) u_{\tau_{i} \tau_{j}}=u_{\tau_{i}} u_{x \tau_{j}}-u_{\tau_{j}} u_{x \tau_{i}} .
$$

Это дифференциальное уравнение (ср. с работами [1]) представляет собой рассматриваемую задачу в упрощенном варианте в том смысле, что аналитичность решения по параметру здесь не важна. Частные решения данного уравнения приведены в разделе 4. Очевидно, что введение новой независимой переменной $\tau_{k}$, отвечающей $\mu_{k}$, приводит к уравнению, совместному с (1.3). Некоторые обобщения, обладающие аналогичными симметрийными свойствами, обсуждаются в приложении Б. Было бы интересно сравнить эти примеры с классификационными результатами, полученными в работах [2] в рамках метода гидродинамических редукций.

Итак, отличительной чертой уравнения (1.1) являются его симметрийные свойства. В случае полиномиальных по $\lambda$ решений достаточный набор симметрий позволяет полностью проинтегрировать уравнение (1.1). Общее решение в случае многочленов $n$-й степени содержит $n$ произвольных функций одного переменного $\kappa_{i}(\lambda)$, и 
задача сводится к интерполяции многочлена по формулам $\left.G_{x}\right|_{\lambda=\gamma_{i}}=\kappa_{i}\left(\gamma_{i}\right)$ (см. раздел 3$)$. При этом нули $\gamma_{i}$ многочлена $G(\lambda)$ играют роль римановых инвариантов для системы гиперболических уравнений для коэффициентов $G(\lambda)$ (ср. с работой [3]).

В разделе 2 показано, что уравнение (1.1), переписанное в терминах лорановских разложений функции $G(\lambda)$, порождает бесконечную последовательность коммутирующих векторных полей, которую можно интерпретировать как дополнительные симметрии уравнений (1.3).

В приложении А, ориентированном на применение уравнения (1.1) в решении спектральных задач второго порядка

$$
\psi_{x x}=U(x, \lambda) \psi
$$

мы обсудим задачу о построении $G(x, \lambda)$ для широкого класса потенциалов $U(x, \lambda)$. Отказываясь от требования полиномиальности по $\lambda$ как функции $G(x, \lambda)$, так и потенциала $U(x, \lambda)$, удается установить между ними взаимно однозначное соответствие. В прямой задаче для этого используется уравнение Риккати, а решение обратной задачи определяет потенциал $U(x, \lambda)$ как производную Шварца по $x$ от $1 / G(x, \lambda)$.

Уравнение (1.1) допускает не только полиномиальные, но и рациональные по спектральному параметру $\lambda$ решения. Обобщения уравнений Дубровина на этот случай и исследование возможных приложений этих решений к уравнениям типа Кортевега-де Фриза (КдФ) представляется одной из интересных нерешенных задач.

Мы закончим введение примером, демонстрирующим существенность выбора подходящей аналитической структуры $G(\lambda)$ (многочлены, мероморфные функции).

ПримеР 1. Уравнение (1.1) допускает редукцию

$$
G=a(\tau, \lambda) x^{2}+2 b(\tau, \lambda) x+c(\tau, \lambda),
$$

приводящую к системе

$$
\frac{d a}{d \tau}=2\{a, b\}, \quad \frac{d c}{d \tau}=2\{b, c\}, \quad \frac{d b}{d \tau}=\{a, c\}, \quad\{f, g\} \stackrel{\text { def }}{=} \frac{f(\mu) g(\lambda)-g(\mu) f(\lambda)}{\lambda-\mu} .
$$

Нетрудно видеть, что дискриминант $b^{2}-a c$ не зависит от $\tau$. Уравнение (1.3) переходит в матричное уравнение

$$
\left(\mu_{i}-\mu_{j}\right) S_{\tau_{i} \tau_{j}}=\left[S_{\tau_{i}}, S_{\tau_{j}}\right], \quad S=\left(\begin{array}{cc}
b & a \\
-c & -b
\end{array}\right) \in s l_{2},
$$

обладающее частными интегралами $\partial_{\tau_{j}}\left(S_{\tau_{i}}, S_{\tau_{i}}\right)=0$, где $\left(S_{1}, S_{2}\right)=\operatorname{tr} S_{1} S_{2}$.

$\mathrm{B}$ частности, при $a=0$ уравнение сводится к линейному:

$$
\frac{d f(\lambda)}{d t}=\frac{f(\lambda)-f(\mu)}{\lambda-\mu}, \quad \frac{d}{d \tau}=2 b(\mu) \frac{d}{d t}, \quad G(\tau, \lambda)=2 b(\lambda)(x+f(\tau, \lambda)) .
$$




\section{2. КОММУТИРУЮЩИЕ ВЕКТОРНЫЕ ПОЛЯ}

Рассматривая локально-аналитические функции, будем считать, что они не имеют существенно особой точки на бесконечности и регулярны в нуле (что можно обеспечить сдвигом $\lambda$ ), так что можно записать разложения вида

$$
G(\lambda)= \begin{cases}A(\lambda)=a_{0} \lambda^{m}+a_{1} \lambda^{m-1}+a_{2} \lambda^{m-2}+\cdots, & \lambda \rightarrow \infty \\ B(\lambda)=b_{0}+b_{1} \lambda+b_{2} \lambda^{2}+\cdots, & \lambda \rightarrow 0\end{cases}
$$

сходящиеся соответственно при $|\lambda|>\rho_{1}$ и $|\lambda|<\rho_{2}$.

Выбрав точку $\mu$ в области сходимости одного из степенны́х рядов (2.1), перепишем уравнение (1.1) в виде

$$
(\lambda-\mu) D_{\tau} A(\lambda)=\langle A(\lambda), G(\mu)\rangle .
$$

Приравняв нулю коэффициент при $\lambda^{m+1}$, получаем $D_{\tau} a_{0}=0$. Делая замену вида $\partial_{x} \rightarrow \phi(x) \partial_{x}, G \rightarrow G / \phi(x)$, можно положить $a_{0}=1$, не теряя общности. Тогда

$$
D_{\tau} a_{n}=\left\langle A_{n}(\mu), G(\mu)\right\rangle, \quad A_{n}(\lambda)=\lambda^{n}+a_{1} \lambda^{n-1}+a_{2} \lambda^{n-2}+\cdots+a_{n} .
$$

Аналогично из уравнения

$$
(\lambda-\mu) D_{\tau} B(\lambda)=\langle B(\lambda), G(\mu)\rangle
$$

в окрестности $\lambda=0$ получаем

$$
D_{\tau} b_{n}=\left\langle A_{-n-1}(\mu), G(\mu)\right\rangle, \quad A_{-1}(\mu)=-\frac{b_{0}}{\mu}, \quad A_{-2}(\mu)=-\frac{b_{0}}{\mu^{2}}-\frac{b_{1}}{\mu}, \quad \ldots
$$

Таким образом, разложения (2.1) и уравнение (1.1) приводят к бесконечной последовательности полиномов $A_{n}$ :

$$
A_{n}(\lambda)=\lambda A_{n-1}(\lambda)+\left\{\begin{array}{ll}
a_{n}, & n>0, \\
b_{-n}, & n<0,
\end{array} \quad A_{0}=1, \quad A_{-1}(\lambda)=-\frac{b_{0}}{\lambda} .\right.
$$

Полиномы $A_{n}, n \in \mathbb{Z}$, и теорема 1 позволяют переписать исходное уравнение (1.1) в виде системы уравнений для коэффициентов рядов $A(\lambda)$ и $B(\lambda)$. Для этого, ориентируясь на работы [1], введем последовательность векторных полей $\mathcal{L}_{n}, n \in \mathbb{Z}$, в виде дифференциальных операторов

$$
\mathcal{L}_{n}=D_{n}-A_{n}(\lambda) D_{0}, \quad D_{n}=\frac{\partial}{\partial t_{n}}, \quad D_{0}=\frac{\partial}{\partial x}
$$

Соответствующая иерархия времен обозначается далее $\mathbf{t}=\left(\ldots, t_{-1}, t_{0}, t_{1}, \ldots\right)$.

Исходя из полученных выше формул для $D_{\tau} a_{n}$ и $D_{\tau} b_{n}$, можно проверить, что коммутативность дифференцирований из теоремы 1 приводит к коммутационным соотношениям

$$
\left[\mathcal{L}_{m}, \mathcal{L}_{n}\right]=0 \quad \Leftrightarrow \quad D_{m} A_{n}-D_{n} A_{m}=\left\langle A_{m}, A_{n}\right\rangle
$$


для всех $m, n \in \mathbb{Z}$. Наконец, эти соотношения для многочленов (2.3) можно переписать как уравнение непосредственно для $G(\lambda)$ :

$$
D_{n} G(\lambda)=\left\langle A_{n}(\lambda), G(\lambda)\right\rangle, \quad G(\lambda)=G(\mathbf{t}, \lambda), \quad n \in \mathbb{Z} .
$$

Эквивалентность (2.5) и (2.6) доказывается, следуя стандартной схеме [4].

Подставив в уравнение (2.6) разложения (2.1), получаем

$$
D_{n} A(\lambda)=\left\langle A_{n}(\lambda), A(\lambda)\right\rangle, \quad D_{n} B(\lambda)=\left\langle A_{n}(\lambda), B(\lambda)\right\rangle, \quad n \in \mathbb{Z} .
$$

В частности, для первого “отрицательного" времени $t_{-1}$ имеем (ср. с работой [5])

$$
D_{-1} A(\lambda)=\frac{b_{0, x}}{\lambda}+\frac{\left\langle a_{1}, b_{0}\right\rangle}{\lambda^{2}}+\frac{\left\langle a_{2}, b_{0}\right\rangle}{\lambda^{3}}+\cdots, \quad D_{-1} B(\lambda)=\left\langle b_{1}, b_{0}\right\rangle+\left\langle b_{2}, b_{0}\right\rangle \lambda+\cdots,
$$

что приводит к бесконечной автономной системе уравнений для переменных $b_{i}$ :

$$
D_{-1} b_{0}=\left\langle b_{1}, b_{0}\right\rangle, \quad D_{-1} b_{1}=\left\langle b_{2}, b_{0}\right\rangle, \quad \ldots,
$$

соответствующей выбору $\mu$ в области сходимости ряда $B(\lambda)$.

Аналогично для первого “положительного" времени $D_{1}=\partial_{t_{1}}$ определяющие уравнения (2.6) дают

$$
D_{1} A(\lambda)=\left\langle\lambda+a_{1}, \frac{a_{2}}{\lambda^{2}}+\frac{a_{3}}{\lambda^{3}}+\cdots\right\rangle, \quad D_{1} B(\lambda)=\left\langle a_{1}, b_{0}\right\rangle+\left(b_{0, x}+\left\langle a_{1}, b_{1}\right\rangle\right) \lambda+\cdots,
$$

что сводится к бесконечной системе уравнений для переменных $a_{i}$ :

$$
D_{1} a_{1}=a_{2, x}, \quad D_{1} a_{2}=a_{3, x}+\left\langle a_{1}, a_{2}\right\rangle, \quad D_{1} a_{3}=a_{4, x}+\left\langle a_{1}, a_{3}\right\rangle, \quad \ldots,
$$

соответствующей выбору $\mu$ в области сходимости ряда $A(\lambda)$.

\section{3. ПОЛИНОМИАЛЬНЫЕ РЕШЕНИЯ}

В полиномиальном случае $G=\lambda^{n}+g_{1} \lambda^{n-1}+\cdots+g_{n}$ бесконечная последовательность времен $\mathbf{t}$ и отвечающих им полиномов (2.3) сводится к конечному базису

$$
D_{i}(G)=\left\langle G_{i}, G\right\rangle, \quad G_{i}=\lambda^{i}+g_{1} \lambda^{i-1}+\cdots+g_{i} .
$$

Данное дифференцирование, отвечающее произвольному $\mu$, можно разложить по этому базису. В частности, $\partial_{x}=D_{0}$, а значению $\mu=0$ отвечает дифференцирование $D_{n-1}$. В последнем случае уравнения для коэффициентов имеют вид

$$
g_{1, t_{n-1}}=g_{n, x}, \quad g_{2, t_{n-1}}=\left\langle g_{1}, g_{n}\right\rangle, \quad \ldots, \quad g_{n, t_{n-1}}=\left\langle g_{n-1}, g_{n}\right\rangle .
$$

Эта система имеет довольно много приложений. Например, случай $n=2$ :

$$
g_{1, t_{1}}=g_{2, x}, \quad g_{2, t_{1}}=\left\langle g_{1}, g_{2}\right\rangle \quad \Leftrightarrow \quad u_{t_{1} t_{1}}=\left\langle u_{x}, u_{t_{1}}\right\rangle \text {, }
$$

описывает модель Чаплыгина в газовой динамике.

2 Теоретическая и математическая физика, т. 153, № 1, 2007 г. 
В общем случае, введя в качестве динамических переменных нули $G(\lambda)$ :

$$
G(\lambda)=\left(\lambda-\gamma_{1}\right)\left(\lambda-\gamma_{2}\right) \cdots\left(\lambda-\gamma_{n}\right),
$$

и полагая $\lambda=\gamma_{i}$ в (1.1), получаем

$$
\left.D_{\tau} G(\lambda)\right|_{\lambda=\gamma_{i}}=\left.\frac{G(\mu) G_{x}(\lambda)}{\mu-\lambda}\right|_{\lambda=\gamma_{i}} \Leftrightarrow \quad D_{\tau} \gamma_{i}=\frac{G(\mu)}{\mu-\gamma_{i}} \gamma_{i, x}, \quad i=1, \ldots, n,
$$

или

$$
D_{\tau} \gamma_{1}=\left(\mu-\gamma_{2}\right) \cdots\left(\mu-\gamma_{n}\right) \gamma_{1, x}, \quad \ldots, \quad D_{\tau} \gamma_{n}=\left(\mu-\gamma_{1}\right) \cdots\left(\mu-\gamma_{n-1}\right) \gamma_{n, x} .
$$

Сравнивая эту формулу с (3.1) при $\lambda=\gamma_{i}$, получаем

$$
D_{\tau}=\mu^{n-1} D_{0}+\mu^{n-2} D_{1}+\cdots+D_{n-1} .
$$

Задача интегрирования (3.3) допускает различные постановки, и мы строим общее решение, ориентируясь на работу [3]. В силу теоремы 1 и формулы (3.4) дифференцирования $D_{i}$ коммутируют. Отождествляя $g_{1}, \ldots, g_{n}$ с элементарными симметрическими многочленами

$$
g_{1}=-\sum \gamma_{k}, \quad g_{2}=\sum_{k<l} \gamma_{k} \gamma_{l}, \quad g_{3}=-\sum_{k<l<m} \gamma_{k} \gamma_{l} \gamma_{m},
$$

и вводя обозначения

$$
g_{i, j}=\left.g_{i}\right|_{\gamma_{j}=0} \quad \Rightarrow \quad g_{1,1}=-\gamma_{2}-\cdots-\gamma_{n}, \quad \ldots, \quad g_{n-1, n}=(-1)^{n} \gamma_{1} \cdots \gamma_{n-1},
$$

приходим к следующему утверждению.

УтВеРЖДЕНИЕ 1. Интегрирование уравнений (3.3) эквивалентно интегрированию совместной системы из $n(n-1)$ уравнений

$$
D_{i}\left(\gamma_{j}\right)=g_{i, j} D_{0}\left(\gamma_{j}\right), \quad i=1, \ldots, n-1, \quad j=1, \ldots, n .
$$

Можно добавить, что уравнение (3.5) с номером $(i, j)$ получается из соответствующего уравнения (3.1) при подстановке $\lambda=\gamma_{j}$, и

$$
g_{i, j}=G_{i}\left(\gamma_{j}\right)=\gamma_{j}^{i}+\gamma_{j}^{i-1} g_{1}+\cdots+g_{i}=-\gamma_{j}^{i-n}\left(\gamma_{j}^{n-i-1} g_{i+1}+\cdots+g_{n}\right), \quad i<n .
$$

Заметим, что нули $\gamma_{j}$ являются инвариантами Римана, т.е. приводят любую из рассматриваемых $n-1$ квазилинейных систем уравнений первого порядка (3.1) (напомним, что (3.2) отвечает $i=n-1)$ к диагональному виду.

Теорема 2. Пусть $D_{i}=\partial_{t_{i}}, i=0, \ldots, n-1$. Тогда общее решение переопределенной системы (3.5) задается формулой

$$
\left[\begin{array}{c}
d t_{0} \\
d t_{1} \\
\vdots \\
d t_{n-1}
\end{array}\right]=\left[\begin{array}{cccc}
\gamma_{1}^{n-1} & \gamma_{2}^{n-1} & \ldots & \gamma_{n}^{n-1} \\
\gamma_{1}^{n-2} & \gamma_{2}^{n-2} & \ldots & \gamma_{n}^{n-2} \\
\vdots & \vdots & \ddots & \vdots \\
1 & 1 & \ldots & 1
\end{array}\right]\left[\begin{array}{c}
\kappa_{1}^{-1}\left(\gamma_{1}\right) d \gamma_{1} \\
\kappa_{2}^{-1}\left(\gamma_{2}\right) d \gamma_{2} \\
\vdots \\
\kappa_{n}^{-1}\left(\gamma_{n}\right) d \gamma_{n}
\end{array}\right]
$$


ДокАЗАТЕльство. Допустим в дополнение к (3.5), что динамика нулей по $x$ определена системой

$$
\gamma_{1, x}=R_{1}\left(\gamma_{1}, \ldots, \gamma_{n}\right), \quad \ldots, \quad \gamma_{n, x}=R_{n}\left(\gamma_{1}, \ldots, \gamma_{n}\right),
$$

и найдем вид функций $R_{i}$ из условий совместности с $(3.5)$, вычисляя перекрестные производные. Например, используя (3.3), находим

$$
\begin{aligned}
D_{0} \gamma_{1} & =R_{1}, \quad D_{\tau} \gamma_{1}=\left(\mu-\gamma_{2}\right) \cdots\left(\mu-\gamma_{n}\right) R_{1} \Rightarrow \\
& \Rightarrow\left[D_{\tau}, D_{0}\right]\left(\gamma_{1}\right)=D_{\tau} R_{1}-D_{0}\left(\mu-\gamma_{2}\right) \cdots\left(\mu-\gamma_{n}\right) R_{1} .
\end{aligned}
$$

Полагая $\mu=\gamma_{2}, \ldots, \gamma_{n}$ в условии совместности $\left[D_{\tau}, D_{0}\right]\left(\gamma_{1}\right)=0$, получаем

$$
\frac{\partial \ln R_{1}}{\partial \gamma_{j}}=\frac{1}{\gamma_{1}-\gamma_{j}}, \quad \frac{\partial}{\partial \gamma_{j}} \ln \left(R_{1} \prod_{j=2}^{n}\left(\gamma_{1}-\gamma_{j}\right)\right)=0, \quad j=2, \ldots, n .
$$

Аналогично остальные условия совместности дают

$$
\frac{\partial \ln R_{i}}{\partial \gamma_{j}}=\frac{1}{\gamma_{i}-\gamma_{j}}, \quad i \neq j, \quad \Rightarrow \quad R_{i}=\frac{\kappa_{i}\left(\gamma_{i}\right)}{\prod^{\prime}\left(\gamma_{i}-\gamma_{j}\right)},
$$

где $n$ функций $\kappa_{1}\left(\gamma_{1}\right), \ldots, \kappa_{n}\left(\gamma_{n}\right)$ произвольны.

Очевидно, что найденная дополнительная динамическая система

$$
\gamma_{1, x}=\frac{\kappa_{1}\left(\gamma_{1}\right)}{\left(\gamma_{1}-\gamma_{2}\right) \cdots\left(\gamma_{1}-\gamma_{n}\right)}, \quad \gamma_{2, x}=\frac{\kappa_{2}\left(\gamma_{2}\right)}{\left(\gamma_{2}-\gamma_{1}\right)\left(\gamma_{2}-\gamma_{3}\right) \cdots\left(\gamma_{2}-\gamma_{n}\right)}, \quad \cdots
$$

вместе с (3.5) явно определяет матрицу Якоби размера $n \times n$

$$
J=\frac{\partial\left(\gamma_{1}, \ldots, \gamma_{n}\right)}{\partial\left(t_{0}, \ldots, t_{n-1}\right)}
$$

из частных производных $n$ нулей по $n$ независимым переменным $\left(t_{0}=x\right)$. Можно непосредственно проверить, что $J$ совпадает с обратной к матрице Вандермонда $W$ из формулы (3.6) с точностью до умножения слева на диагональную матрицу:

$$
J=\operatorname{diag}\left(\kappa_{1}\left(\gamma_{1}\right), \kappa_{2}\left(\gamma_{2}\right), \ldots, \kappa_{n}\left(\gamma_{n}\right)\right) W^{-1} .
$$

Остается заметить, что формула (3.6), записанная в виде

$$
d t_{n-1}=\frac{d \gamma_{1}}{\kappa_{1}\left(\gamma_{1}\right)}+\cdots+\frac{d \gamma_{n}}{\kappa_{n}\left(\gamma_{n}\right)}, \quad \ldots, \quad d x=\frac{\gamma_{1}^{n-1} d \gamma_{1}}{\kappa_{1}\left(\gamma_{1}\right)}+\cdots+\frac{\gamma_{n}^{n-1} d \gamma_{n}}{\kappa_{n}\left(\gamma_{n}\right)},
$$

сводит задачу определения зависимости $t_{0}, \ldots, t_{n-1}$ от $\gamma_{1}, \ldots, \gamma_{n}$ к квадратурам.

Очевидно, что многочлен $G_{x}(\lambda)$ имеет степень $(n-1)$, и из формул $(3.8)$ и соотношения

$$
G_{x}(\lambda)=-\gamma_{1, x}\left(\lambda-\gamma_{2}\right) \cdots\left(\lambda-\gamma_{n}\right)-\cdots-\gamma_{n, x}\left(\lambda-\gamma_{1}\right) \cdots\left(\lambda-\gamma_{n-1}\right)
$$

следует, что для вычисления якобиана (3.9) и интегрирования дифференциальных уравнений (3.1) достаточно задать произвольным образом функции

$$
\left.G_{x}(\lambda)\right|_{\lambda=\gamma_{j}}=-\kappa_{j}\left(\gamma_{j}\right), \quad j=1, \ldots, n .
$$


СлеДСтвиЕ. Теорема 2 позволяет определить все производные $D_{i}(G)$ в терминах $\gamma_{1}, \ldots, \gamma_{n}$ явно, без интегрирования дифференииальных уравнений (3.1). В частности, $D_{0}(G)=G_{x}$ определяется при помощи интерполяиионной формуль Лагранжа:

$$
G_{x}(\lambda)=-\kappa_{1}\left(\gamma_{1}\right) \frac{\left(\lambda-\gamma_{2}\right) \cdots\left(\lambda-\gamma_{n}\right)}{\left(\gamma_{1}-\gamma_{2}\right) \cdots\left(\gamma_{1}-\gamma_{n}\right)}-\cdots-\kappa_{n}\left(\gamma_{n}\right) \frac{\left(\lambda-\gamma_{1}\right) \cdots\left(\lambda-\gamma_{n-1}\right)}{\left(\gamma_{n}-\gamma_{1}\right) \cdots\left(\gamma_{n}-\gamma_{n-1}\right)} .
$$

Вместо независимой переменной $t_{n-1}$, соответствующей $\mu=0$, можно использовать $\tau$. Для перехода от переменных $t_{0}=x, t_{1}, \ldots, t_{n-1}$ к переменным $t_{0}, t_{1}, \ldots$, $t_{n-2}, \tau$ нужен якобиан

$$
\frac{\partial\left(t_{0}, \ldots, \tau\right)}{\partial\left(\gamma_{1}, \ldots, \gamma_{n}\right)}=\frac{\partial\left(t_{0}, \ldots, \tau\right)}{\partial\left(t_{0}, \ldots, t_{n-1}\right)} \frac{\partial\left(t_{0}, \ldots, t_{n-1}\right)}{\partial\left(\gamma_{1}, \ldots, \gamma_{n}\right)} .
$$

Так как $d \tau=\mu^{n-1} d t_{0}+\mu^{n-2} d t_{1}+\cdots+d t_{n-1}$, то

$$
d \tau=\sum \frac{\kappa\left(\gamma_{i}\right) d \gamma_{i}}{\kappa_{i}\left(\gamma_{i}\right)}, \quad \kappa(\gamma)=(\mu \gamma)^{n-1}+(\mu \gamma)^{n-2}+\cdots+1
$$

Для применения теоремы 2 к уравнению (1.3) достаточно теперь выразить потенциал через нули $\gamma_{j}(x, \alpha, \beta), j=1,2 \ldots$ :

$$
d u=\frac{\gamma_{1}^{n} d \gamma_{1}}{\kappa_{1}\left(\gamma_{1}\right)}+\cdots+\frac{\gamma_{n}^{n} d \gamma_{n}}{\kappa_{n}\left(\gamma_{n}\right)} \quad \Rightarrow \quad D_{j} u=g_{j}
$$

\section{4. ОТОБРАЖЕНИЕ $G \rightarrow U$}

Рассмотрим классификацию полиномиальных решений $G(\lambda)$ уравнения (1.1), использующую следующее отображение:

$$
G \rightarrow U: 4 U=\frac{K(\lambda)}{G^{2}}-\frac{G_{x}^{2}}{G^{2}}+2 \frac{G_{x x}}{G} .
$$

Отметим, что рассматриваемое отображение $G \rightarrow U$ имеет нетривиальное ядро, состоящее из квадратичных по $x$ полиномов (1.4) (см. пример 1). Действительно, умножение уравнения (4.1) на $G^{2}$ дает после дифференцирования линейное уравнение третьего порядка

$$
2 U G_{x}+U_{x} G=\frac{1}{2} G_{x x x} .
$$

Полагая в этом уравнении $U=0$, получаем $G_{x x x}=0$. Заметим еще, что в силу однородности формулы (4.1) замена

$$
\widetilde{G}=\kappa(\lambda) G, \quad \widetilde{K}=\kappa^{2} K \Rightarrow \widetilde{U}=U
$$

не меняет $U$. Это позволяет рассматривать полиномиальные решения $\widetilde{G}=\kappa(\lambda) G$ и $G$, отличающиеся зависящим только от $\lambda$ множителем $\kappa(\lambda)$, как эквивалентные.

Использование отображения (4.1) для классификации полиномиальных решений $G(\lambda)$ не в последнюю очередь связано с созданной школой Новикова теорией конечнозонных потенциалов $U$ для спектральных задач второго порядка

$$
\psi_{x x}=U(x, \lambda) \psi
$$


В связи с этими приложениями в спектральной теории полезно иметь в виду следующее соотношение:

$$
f^{ \pm} \stackrel{\text { def }}{=} \frac{G_{x}}{2 G} \pm \frac{\sqrt{K(\lambda)}}{2 G} \Rightarrow f_{x}^{ \pm}+\left(f^{ \pm}\right)^{2}=\frac{K(\lambda)}{4 G^{2}}+\frac{G_{x x}}{2 G}-\frac{G_{x}^{2}}{4 G^{2}}=U,
$$

связывающее уравнение (4.1) с уравнением Риккати $f_{x}+f^{2}=U$. Прямая задача о построении при заданном потенциале $U$ двух специальных решений $f=f^{ \pm}$ уравнения Риккати таких, что

$$
f^{+}-f^{-}=\frac{\sqrt{K(\lambda)}}{G}
$$

обсуждается в приложении А. Это позволяет, в частности, связать функцию $K(\lambda)$ из формулы (4.1) с вронскианом двух решений $\psi^{ \pm}$спектральной задачи (4.3), соответствующих в силу известного правила $\left(\ln \psi^{ \pm}\right)_{x}=f^{ \pm}$этим специальным решениям $f=f^{ \pm}$уравнения Риккати.

Нули $\lambda=\gamma_{i}$ полиномиальных решений $G(\lambda)$ уравнения (1.1) приводят, вообще говоря, к полюсам потенциала, определенного формулой (4.1), что в приложениях приходит в противоречие с аналитической структурой потенциала $U(\lambda)$, заданной априори. Сформулированная ниже теорема указывает, как можно избавиться от этих нежелательных полюсов за счет специального выбора функций $\kappa_{j}(\lambda)$ в формуле (3.11).

ТЕОРема 3. Потенииал $U(\lambda, x)$ как функиия от $\lambda$ не имеет движущихся полюсов $\lambda=\gamma_{j}(x), \gamma_{j, x} \neq 0$, тогда и только тогда, когда выполнены следующие условия регулярности:

$$
\kappa_{i}(\lambda)= \pm \sqrt{K(\lambda)}, \quad i=1, \ldots, n .
$$

ДокАЗАТЕЛЬСтво. Для доказательства мы выведем уравнения, аналогичные (3.8), непосредственно из определяющего уравнения (4.1), используя только условие регулярности и не обращаясь к утверждению 1. С этой целью подставим

$$
\frac{1}{G}=\frac{\varepsilon_{1}}{\gamma_{1}-\lambda}+\cdots+\frac{\varepsilon_{n}}{\gamma_{n}-\lambda}, \quad \Gamma=\frac{G_{x}}{G}=\frac{\gamma_{1, x}}{\gamma_{1}-\lambda}+\cdots+\frac{\gamma_{n, x}}{\gamma_{n}-\lambda}, \quad \varepsilon_{i} \stackrel{\text { def }}{=} \prod_{j \neq i} \frac{1}{\gamma_{i}-\gamma_{j}},
$$

в формулу

$$
4 U=\frac{K(\lambda)}{G^{2}}+2 \Gamma_{x}+\Gamma^{2} .
$$

Приравнивая нулю коэффициенты при

$$
\left(\lambda-\gamma_{1}\right)^{-2}, \quad\left(\lambda-\gamma_{2}\right)^{-2}, \quad \ldots, \quad\left(\lambda-\gamma_{n}\right)^{-2},
$$

получаем $n$ уравнений Дубровина для $\gamma_{1}, \ldots, \gamma_{n}$ :

$$
\gamma_{1, x}=\frac{\sqrt{K\left(\gamma_{1}\right)}}{\left(\gamma_{1}-\gamma_{2}\right) \cdots\left(\gamma_{1}-\gamma_{n}\right)}, \quad \ldots, \quad \gamma_{n, x}=\frac{\sqrt{K\left(\gamma_{n}\right)}}{\left(\gamma_{n}-\gamma_{1}\right) \cdots\left(\gamma_{n}-\gamma_{n-1}\right)} .
$$

Сравнив эти уравнения с (3.8), заключаем, что выполняются условия (4.5). 
Оказывается, эти условия не только необходимы, но и достаточны для регулярности потенциала $U$, определенного формулой (4.1) по решению $G$ уравнений (3.8). Действительно, дифференцируя по $x$ уравнения (4.7), получаем

$$
\gamma_{i, x x}=\gamma_{i, x}^{2}\left(\frac{1}{2} \frac{K^{\prime}}{K}\left(\gamma_{i}\right)-\sum_{j \neq i} \frac{1}{\gamma_{i}-\gamma_{j}}\right)+\sum_{j \neq i} \frac{\gamma_{i, x} \gamma_{j, x}}{\gamma_{i}-\gamma_{j}}, \quad i=1, \ldots, n .
$$

С другой стороны,

$$
\frac{K(\lambda)}{G^{2}(\lambda)}=K_{2}+K_{1}+K_{0}, \quad K_{2}=\sum_{i=1}^{n} \frac{\varepsilon_{i}^{2} K\left(\gamma_{i}\right)}{\left(\lambda-\gamma_{i}\right)^{2}}, \quad K_{1}=\sum_{i=1}^{n} \frac{\varepsilon_{i}^{2} K\left(\gamma_{i}\right) K_{1, i}}{\lambda-\gamma_{i}}
$$

где

$$
K_{1, i}=\left.\frac{d}{d \lambda} \ln K(\lambda)\right|_{\lambda=\gamma_{i}}-2 \sum_{j \neq i} \frac{1}{\gamma_{i}-\gamma_{j}},
$$

а функция $K_{0}$ регулярна. Так как согласно (4.6)

$$
4 U=\frac{K(\lambda)}{G^{2}}-\sum_{i=1}^{n} \frac{\gamma_{i, x}^{2}}{\left(\gamma_{i}-\lambda\right)^{2}}+2 \sum_{i<j} \frac{\gamma_{i, x} \gamma_{j, x}}{\left(\gamma_{i}-\lambda\right)\left(\gamma_{j}-\lambda\right)}+2 \sum_{i=1}^{n} \frac{\gamma_{i, x x}}{\left(\gamma_{i}-\lambda\right)},
$$

то мы получаем, что в силу уравнений Дубровина (4.7) сокращаются полюсы и первого, и второго порядков. Теорема доказана.

В силу вышесказанного, если произвольные априори функции $\kappa_{j}(\lambda)$ в формуле (3.10), задающие решение $G$, удовлетворяют условию регулярности (4.5) с аналитической функцией $K(\lambda)$, то потенциал $U$ аналитичен по $\lambda$. В силу теоремы Лиувилля функция, аналитическая по $\lambda$ во всей расширенной плоскости, является постоянной. В частности (см. отображение (4.1)), полиномиальным функциям $K(\lambda)$ соответствуют полиномиальные по $\lambda$ потенциалы $U$. Очень простая структура потенциала $U$ в этом случае определяется разложением в окрестности $\lambda=\infty$ функции

$$
H(\lambda)=\frac{\lambda^{n}}{G}=1+\frac{h_{1}}{\lambda}+\frac{h_{2}}{\lambda^{2}}+\cdots, \quad h_{1}=-g_{1}, \quad h_{2}=g_{1}^{2}-g_{2}, \quad \ldots,
$$

и коэффициентами $c_{i}$ разложения

$$
\lambda^{-m} K(\lambda)=4\left(1+\frac{c_{1}}{\lambda}+\frac{c_{2}}{\lambda^{2}}+\cdots\right),
$$

где $m$ обозначает порядок полюса $K(\lambda)$ в точке $\lambda=\infty$. Отметим, что коэффициенты разложения (4.10) определяют законы сохранения уравнений (3.11), так как

$$
D_{i}(H)=D_{i}\left(\frac{\lambda^{n}}{G}\right)=\lambda^{n} \frac{G G_{i, x}-G_{x} G_{i}}{G^{2}}=H G_{i, x}+H_{x} G_{i}=D_{0}\left(H G_{i}\right) .
$$

Итак, в полиномиальном случае вид потенциала $U$ определяется первым слагаемым в формуле (4.1) и, если степень $m$ полинома $K(\lambda)$ превосходит степень $2 n$ многочлена $G^{2}(\lambda)$ на 1 или 2 , то

$$
\frac{K(\lambda)}{4 G^{2}}=\left\{\begin{array}{l}
\lambda+2 h_{1}+c_{1}+\cdots, \\
\lambda^{2}+\lambda\left(2 h_{1}+c_{1}\right)+h_{1}^{2}+2 h_{2}+2 c_{1} h_{1}+c_{2}+\cdots,
\end{array}\right.
$$


соответственно. Так как остальные члены в формуле (4.1) обращаются в нуль при $\lambda=\infty$, то в последнем случае при $m=2 n+2$ получаем

$$
U=\lambda^{2}+u_{1} \lambda+u_{2}, \quad u_{1}=2 h_{1}+c_{1}, \quad u_{2}=h_{1}^{2}+2 h_{2}+2 c_{1} h_{1}+c_{2} .
$$

Аналогично в случае $m=2 n+1$

$$
U=\lambda+u(x), \quad u(x)=c_{1}+2 h_{1}=2 \sum_{j=1}^{n} \gamma_{j}(x)-\sum_{i=1}^{2 n+1} e_{i},
$$

где $e_{i}, i=1,2, \ldots$, обозначают $2 n+1$ нуль многочлена $K(\lambda)$. Как известно [6], в вещественном случае для регулярности потенциала на всей оси необходимо и достаточно, чтобы начальные условия для $\gamma_{i}$ лежали в запрещенных зонах спектра:

$$
e_{1}<e_{2}<g_{1}<e_{3}<\cdots<e_{2 n}<\gamma_{n}<e_{2 n+1} .
$$

Если, например, в дополнение к полюсу порядка $m=2 n+1$ на бесконечности функция $K(\lambda)$ имеет полюс первого порядка в точке $\lambda=0$, то формула (4.1) определяет потенциал в виде

$$
U=\lambda+u+\frac{v}{\lambda}=\lambda+2 h_{1}+c_{1}+\frac{1}{\lambda}\left(h_{1}^{2}+2 h_{2}-\frac{1}{2} h_{1, x x}\right) .
$$

В этом случае близкую к изложенной выше схему построения частных решений можно найти в работе [7] (см. также работу [4]).

Дополнительные возможности классификации полиномиальных решений $G(\lambda)$ возникают, если заметить, что отображение (4.1) можно факторизовать. Для этого достаточно ввести промежуточное отображение $G \rightarrow V$, определенное формулой

$$
V=\frac{G_{x}+\kappa(\lambda)}{2 G}
$$

Тогда в силу формулы (4.4)

$$
U=V_{x}+V^{2}, \quad K(\lambda)=\kappa^{2}(\lambda) .
$$

При этом условие регулярности $V$ эквивалентно в силу формулы (3.11) соотношениям $\kappa_{i}=-\kappa, i=1, \ldots, n$ (ср. с условиями (4.5)). Как и в случае (4.1), условие регулярности гарантирует, что если $\kappa(\lambda)$ - многочлен, то $V$ - тоже многочлен. В частности, выбрав в формуле полином $\kappa(\lambda)$ степени $m=n+1$ мы, аналогично выражению (4.12), находим

$$
V=\lambda+v(x), \quad v=-g_{1}, \quad U=\lambda^{2}+2 v \lambda+v^{2}+v_{x} .
$$

Для иллюстрации многообразия приложений условий регулярности типа (4.5) и теоремы 2 ниже приводится список скалярных уравнений с частными производными, соответствующих уравнениям (3.1) в случаях (4.12) и (4.15). Так как аналитическая структура $U$ и $V$ в нашем случае существенно проще структуры $G$, естественно переписать уравнения (3.1) в терминах $U$ и $V$. Для этого достаточно заметить, что

$$
G_{t}=R G_{x}-G R_{x} \Rightarrow V_{t}=\left(R V-\frac{1}{2} R_{x}\right)_{x}
$$


и аналогично

$$
G_{t}=R G_{x}-G R_{x} \quad \Leftrightarrow \quad f_{t}=\left(R f-\frac{1}{2} R_{x}\right)_{x} \quad \Rightarrow \quad U_{t}=2 U R_{x}+U_{x} R-\frac{1}{2} R_{x x x} .
$$

Пример 2. Выбрав $R=G_{1}=\lambda+g_{1}$, очевидным образом получаем в случае (4.15), (4.16) уравнение Бюргерса

$$
v_{t}=v_{x x}+2 v v_{x}=\left[\left(\lambda+g_{1}\right)(\lambda+v)-g_{1, x}\right]_{x},
$$

а в случае (4.12), (4.17) - уравнение КдФ

$$
4 u_{t}=u_{x x x}-6 u u_{x}+\varepsilon u_{x} .
$$

С другой стороны, используя в (4.17) уравнение для $f$, нетрудно получить модифицированное уравнение КдФ. Действительно, второе из уравнений (4.17) при $R=G_{1}=\lambda+g_{1}$ дает

$$
D_{1}(f)=D_{x}\left(\lambda f+f g_{1}-\frac{1}{2} g_{1, x}\right), \quad f_{x}+f^{2}=U=\lambda-2 g_{1}+c_{1} .
$$

Выразив $g_{1}$ через $f$ и подставив в первое из рассматриваемых уравнений, мы получаем

$$
4 f_{t}=D_{x}\left(f_{x x}-2 f^{3}+6 \lambda f\right)+\varepsilon f_{x} .
$$

Связь между решениями уравнений (4.19), (4.20) задает зависящее от $\lambda$ преобразование Миуры $u=f_{x}+f^{2}+\lambda$.

Наконец, как мы сейчас покажем, исходная система уравнений (3.2) приводит в случае $U=\lambda+u$ прямо к sh-уравнению

$$
\varphi_{x \tau}=\frac{1}{2} k_{1} e^{-\varphi}-2 e^{\varphi}, \quad e^{\varphi}=G(0), \quad k_{1}=\left.\frac{d K}{d \lambda}\right|_{\lambda=0}, \quad K(0)=0 .
$$

Действительно, из (3.2), (4.1) мы имеем соответственно

$$
g_{\tau}=\langle\tilde{g}, g\rangle=\tilde{g} g_{x}-g \tilde{g}_{x}, \quad 4(\lambda+u) G^{2}=K(\lambda)-G_{x}^{2}+2 G_{x x} G,
$$

где введены обозначения

$$
g=G(0), \quad \tilde{g}=\left.\frac{d G}{d \lambda}\right|_{\lambda=0} .
$$

Продифференцировав по $\lambda$ второе из уравнений (4.22) и исключив после этого $u$, находим

$$
2 g^{2}-\frac{1}{2} k_{1}+k_{0} \frac{\tilde{g}}{g}+\langle\tilde{g}, g\rangle_{x}-(\ln g)_{x}\langle\tilde{g}, g\rangle=0, \quad k_{0} \stackrel{\text { def }}{=} K(0) .
$$

Полагая $k_{0}=0$ и заменяя $\langle\tilde{g}, g\rangle$ на $g_{\tau}$, находим после очевидных преобразований, что последнее уравнение сводится к (4.21). 
В заключение мы рассмотрим кратко приложения уравнения (1.1) к вопросу об интегрируемости уравнений (4.17), (4.16) типа рассмотренных в примере 2 при замене полиномиальных решений решениями $G(\lambda)$ вида (2.1). Заметив, что при возрастании степени полиномов $G(\lambda)$ решения, соответствующие степени $n$, автоматически остаются решениями системы (3.1) при замене $n$ на $n+1$, естественно рассчитывать, что многие формулы предыдущего раздела, включая (4.4)-(4.17), остаются справедливыми и в общем случае (2.1). Что это действительно так, следует из сравнения уравнений (3.1) и (2.6) при замене $G(\lambda) \rightarrow A(\lambda)$, где

$$
A=1+\sum_{j=1}^{\infty} \lambda^{-j} a_{j}=1+\frac{a_{1}}{\lambda}+\frac{a_{2}}{\lambda^{2}}+\cdots .
$$

Основное отличие рассматриваемых уравнений

$$
D_{n} A(\lambda)=\left\langle A_{n}(\lambda), A(\lambda)\right\rangle, \quad n=1,2,3, \ldots,
$$

относительно коэффициентов ряда (4.23) от соответствующих уравнений (3.1) заключается в возникающей здесь проблеме замыкания бесконечной цепочки уравнений вида $(2.8)^{1)}$. Проблемам, возникающим в связи с заменой условий регулярности условиями обрыва рядов

$$
U=\lambda^{m}+\sum_{j \geqslant 1} \lambda^{m-j} u_{j}, \quad V=\lambda^{m^{\prime}}+\sum_{j \geqslant 1} \lambda^{m^{\prime}-j} v_{j},
$$

полученных из (4.23) в результате отображений (4.1) и (4.14), посвящен цикл работ [1]. Там же можно найти интересные примеры нестандартных замыканий уравнений (4.24).

\section{Уравнение Риккати}

ПРИЛОЖКНИЕ А

Чтобы установить связь отображения (4.1) и формулы (4.4) с производной Шварца и уравнением Риккати, докажем следующее элементарное утверждение.

УТВЕРЖДЕНИЕ П.2. Пусть $\psi_{1}, \psi_{2}-$ два линейно независимых решения уравнения второго порядка $\psi_{x x}=U \psi$. Тогда функиии

$$
A_{1}=\psi_{1}^{2}, \quad A_{2}=\psi_{2}^{2}, \quad A_{3}=\psi_{1} \psi_{2}
$$

образуют базис пространства решений уравнения третвего порядка (ср. с уравнением (4.2))

$$
A_{x x x}=4 U A_{x}+2 U_{x} A .
$$

Более того, функиия $\varphi=\psi_{1} / \psi_{2}$ удовлетворяет уравнению Шварца

$$
\frac{3 \varphi_{x x}^{2}}{4 \varphi_{x}^{2}}-\frac{\varphi_{x x x}}{2 \varphi_{x}}=U(x)
$$

\footnotetext{
1) С алгебраической точки зрения вопрос о замыкании этих систем сводится, по-видимому, к выделению соответствующих подагебр алгебры (2.5).
} 
а функция $A=\psi_{1} \psi_{2}-$ уравнению

$$
4 U(x) A^{2}+A_{x}^{2}-2 A A_{x x}=w^{2},
$$

где $w$ - вронскиан $w=\left\langle\psi_{1}, \psi_{2}\right\rangle=\psi_{1} \psi_{2, x}-\psi_{1, x} \psi_{2}$.

ДокАЗАтЕЛЬСтво. Вычислим вронскиан функций $A_{1}, A_{2}, A_{3}$ :

$$
W=\left\langle A_{1}, A_{2}, A_{3}\right\rangle=\left(\psi_{1} \psi_{2, x}-\psi_{2} \psi_{1, x}\right)^{3}=\left\langle\psi_{1}, \psi_{2}\right\rangle^{3} .
$$

Следовательно, $W=$ const $\neq 0$, и функции $A_{i}$ линейно независимы. Введя обозначения

$$
\varphi=\frac{\psi_{1}}{\psi_{2}}, \quad f_{j}=\frac{\psi_{j, x}}{\psi_{j}},
$$

нетрудно получить, что

$$
\varphi_{x}=\frac{\left\langle\psi_{2}, \psi_{1}\right\rangle}{\psi_{2}^{2}}=\frac{w}{\psi_{2}^{2}}, \quad \frac{\varphi_{x x}}{\varphi_{x}}=-2 \frac{\psi_{2 x}}{\psi_{2}}=-2 f_{2}
$$

и, таким образом, уравнение (П.1) является следствием уравнения Риккати $f_{2, x}+$ $f_{2}^{2}=U$

Далее, нетрудно проверить, что

$$
\frac{w}{A_{3}}=f_{2}-f_{1}, \quad \frac{A_{3, x}}{A_{3}}=f_{2}+f_{1},
$$

откуда следует, что

$$
f_{1}=\frac{A_{3, x}-w}{2 A_{3}}, \quad f_{2}=\frac{A_{3, x}+w}{2 A_{3}} .
$$

Подстановка этих выражений для $f_{j}$ в уравнение Риккати приводит к уравнению (П.2) с $A=A_{3}$, и последующее дифференцирование по $x$ дает уравнение (4.2). Аналогично доказывается, что $A_{1}, A_{2}$ также удовлетворяют уравнению (4.2); в этих случаях $w=0$ в (П.2).

Записав уравнение (П.2) в виде

$$
4 U+\frac{A_{x}^{2}}{A^{2}}-\frac{2 A_{x x}}{A}=\frac{w^{2}}{A^{2}}
$$

и обозначив $H=1 / A$, находим, что

$$
U=\frac{3 H_{x}^{2}}{4 H^{2}}-\frac{H_{x x}}{2 H}+w^{2} H^{2} .
$$

Сравнив это уравнение с уравнением Шварца (П.1), обнаруживаем немедленно, что оно отвечает частному случаю (П.3) с $w=0$ и $H=\varphi_{x}$.

Таким образом, мы видим, что замена

$$
F \stackrel{\text { def }}{=} f_{1}-f_{2}=\frac{w}{G}, \quad \frac{3 F_{x}^{2}}{4 F^{2}}-\frac{F_{x x}}{2 F}+\frac{F^{2}}{4}=U
$$


действительно дает уравнение (4.1), использованное для определения отображения $G \rightarrow U$ (см. раздел 4). Учитывая очевидную связь формулы (П.3) с производной Шварца, можно сказать, что этот переход от $G$ к $U$ сводится, в определенном смысле, к вычислению производной Шварца от $F=G^{-1}$.

Переходя к прямой задаче о построении $G$ по заданному априори потенциалу $U$, мы будем считать, что потенциал задан формальным рядом (4.25):

$$
U=\lambda^{m}+\sum_{j \geqslant 1} \lambda^{m-j} u_{j}, \quad m>0 .
$$

Оказывается, что на уровне формальных рядов типа (4.23) исчезают проблемы, возникающие при попытке явно решить уравнение Риккати. Чтобы не загромождать изложение, мы ограничимся частным случаем $m=2$.

Лемма. Уравнение Риккати с потенциалом вида

$$
U(x, k)=\lambda^{2}+\lambda u_{1}(x)+u_{2}(x)+\lambda^{-1} u_{3}+\cdots
$$

имеет ровно два решения $f=f^{ \pm}$в виде формальных степеннъих рядов по $\lambda$ :

$$
f^{+}=\lambda+\frac{1}{2} u_{1}+f_{1}^{+} \lambda^{-1}+\cdots, \quad f^{-}=-\lambda-\frac{1}{2} u_{1}+f_{1}^{-} \lambda^{-1}+\cdots .
$$

ДокАзАтЕЛЬСтво. Подставляя ряд $f=\sum_{j=j_{0}}^{\infty} \lambda^{-j} f_{j}$ в уравнение $f_{x}+f^{2}=$ $U(x, \lambda)$ и собирая коэффициенты при степенях $\lambda$, получаем $\left(f_{-1}^{ \pm}\right)^{2}=1, j_{0}=-1$ и формулы для коэффициентов $f \pm_{0}$. Последующие коэффициенты $f_{j}^{+}$и $f_{j}^{-}$вычисляются по рекуррентным формулам

$$
2 f_{j+1}^{+}+f_{j, x}^{+}+\sum_{j^{\prime}+j^{\prime \prime}=j} f_{j^{\prime}}^{+} f_{j^{\prime \prime}}^{+}=u_{j}, \quad 2 f_{j+1}^{-}=f_{j, x}^{-}+\sum_{j^{\prime}+j^{\prime \prime}=j} f_{j^{\prime}}^{-} f_{j^{\prime \prime}}^{-}-u_{j}, \quad j>1 .
$$

Следовательно, коэффициенты рядов $f_{j}^{ \pm}$являются корректно определенными дифференциальными полиномами от $u_{j}$.

Важно, что коэффициенты $f_{j}^{ \pm}$построенных в лемме степенны́х рядов (П.5) определены однозначно и явно выражаются через коэффициенты потенциала $U=U(x, k)$ и его производных по $x$.

ПРИЛОЖКНИЕ Б

\section{Совместные тройки уравнений}

Уравнение (1.3) представляет собой пример совместной тройки вида

$$
\begin{aligned}
& u_{\xi \eta}=f\left(u, u_{\xi}, u_{\eta}, u_{x}, u_{x \xi}, u_{x \eta}\right), \\
& u_{\xi \zeta}=g\left(u, u_{\xi}, u_{\zeta}, u_{x}, u_{x \xi}, u_{x \zeta}\right), \\
& u_{\eta \zeta}=h\left(u, u_{\eta}, u_{\zeta}, u_{x}, u_{x \eta}, u_{x \zeta}\right),
\end{aligned}
$$

здесь и далее $\left(\tau_{1}, \tau_{2}, \tau_{3}\right)=(\xi, \eta, \zeta)$. Более симметричный пример дает тройка

$$
\left(\mu_{j}-\mu_{i}\right) u_{x} u_{\tau_{i} \tau_{j}}-\mu_{j} u_{\tau_{i}} u_{x \tau_{j}}+\mu_{i} u_{\tau_{j}} u_{x \tau_{i}}=0,
$$


в которой переменная $x$ фактически равноправна с остальными. Отметим, что это уравнение инвариантно относительно замен $u=a(\tilde{u}), x=b(\tilde{x}), \tau_{i}=c_{i}\left(\tilde{\tau}_{i}\right)$ с произвольными функциями, что дает частное решение $u=a\left(b(x) c\left(\tau_{i}\right) d\left(\tau_{j}\right)\right)$.

Уравнения (1.3) и (П.7) тесно связаны. Действительно, (П.7) получается из (1.3) при исключении производных $u_{x \tau_{i}}$ и выборе одного из $\tau_{i}$ в качестве нового $x$. Кроме того, уравнение (П.7) можно переписать в виде

$$
\left(\frac{u_{\tau_{i}}}{\mu_{i} u_{x}}\right)_{\tau_{j}}=\left(\frac{u_{\tau_{j}}}{\mu_{j} u_{x}}\right)_{\tau_{i}},
$$

и подстановка $v_{\tau_{i}}=u_{\tau_{i}} /\left(\mu_{i} u_{x}\right)$ снова приводит к уравнению $(1.3)$ :

$$
\left(\mu_{i}^{-1}-\mu_{j}^{-1}\right) v_{\tau_{i} \tau_{j}}=v_{\tau_{i}} v_{x \tau_{j}}-v_{\tau_{j}} v_{x \tau_{i}} .
$$

Классификация совместных систем вида (П.6) представляется преждевременной задачей, пока не поняты как следует методы их интегрирования. Тем не менее несложный предварительный анализ показывает, что класс таких систем может быть довольно богатым. Допустим, что правые части квазилинейны и не содержат $u$ явно. Тогда имеется семейство совместных троек, определенное следующим образом.

УтВеРЖДЕНИЕ П.3. Пусть $(X, \widetilde{X}),(A, \tilde{A}),(B, \widetilde{B}),(C, \widetilde{C})$ - решения системъ обыкновенных дифференциальных уравнений вида

$$
X^{\prime}=k_{1} X^{2}+k_{2} X \tilde{X}+k_{3} \tilde{X}^{2}, \quad \tilde{X}^{\prime}=k_{4} X^{2}+k_{5} X \tilde{X}+k_{6} \tilde{X}^{2},
$$

причем следующие функиии не равны тождественно нулю:

$$
\begin{aligned}
a\left(u_{\eta}, u_{\zeta}\right) & =B\left(u_{\eta}\right) \widetilde{C}\left(u_{\zeta}\right)-\widetilde{B}\left(u_{\eta}\right) C\left(u_{\zeta}\right), & & p\left(u_{\xi}, u_{x}\right)=A\left(u_{\xi}\right) \widetilde{X}\left(u_{x}\right)-\tilde{A}\left(u_{\xi}\right) X\left(u_{x}\right), \\
b\left(u_{\zeta}, u_{\xi}\right) & =C\left(u_{\zeta}\right) \tilde{A}\left(u_{\xi}\right)-\widetilde{C}\left(u_{\zeta}\right) A\left(u_{\xi}\right), & & q\left(u_{\eta}, u_{x}\right)=B\left(u_{\eta}\right) \widetilde{X}\left(u_{x}\right)-\widetilde{B}\left(u_{\eta}\right) X\left(u_{x}\right), \\
c\left(u_{\xi}, u_{\eta}\right) & =A\left(u_{\xi}\right) \widetilde{B}\left(u_{\eta}\right)-\tilde{A}\left(u_{\xi}\right) B\left(u_{\eta}\right), & & r\left(u_{\zeta}, u_{x}\right)=C\left(u_{\zeta}\right) \widetilde{X}\left(u_{x}\right)-\widetilde{C}\left(u_{\zeta}\right) X\left(u_{x}\right) .
\end{aligned}
$$

Тогда совместны следующие уравнения:

$$
\begin{aligned}
c\left(u_{\xi}, u_{\eta}\right) u_{\xi \eta} & =p\left(u_{\xi}, u_{x}\right) u_{x \xi}-q\left(u_{\eta}, u_{x}\right) u_{x \eta}, \\
a\left(u_{\eta}, u_{\zeta}\right) u_{\eta \zeta} & =q\left(u_{\eta}, u_{x}\right) u_{x \eta}-r\left(u_{\zeta}, u_{x}\right) u_{x \zeta}, \\
b\left(u_{\zeta}, u_{\xi}\right) u_{\zeta \xi} & =r\left(u_{\zeta}, u_{x}\right) u_{x \zeta}-p\left(u_{\xi}, u_{x}\right) u_{x \xi} .
\end{aligned}
$$

Линейные преобразования приводят второе уравнение системы (П.8) к виду $\widetilde{X}^{\prime}=$ $k X \widetilde{X}$ или $\tilde{X}^{\prime}=k \widetilde{X}^{2}$, после чего нетрудно показать, что решение находится в элементарных функциях. В частности, система (П.7) отвечает функциям $A_{i}=\mu_{i} / u_{\tau_{i}}$, $\tilde{A}_{i}=1 / u_{\tau_{i}}, X=\delta / u_{x}, \widetilde{X}=1 / u_{x}$, которые решают систему $X^{\prime}=-X \widetilde{X}, \widetilde{X}^{\prime}=-\widetilde{X}^{2}$. Здесь одной из постоянных интегрирования можно пренебречь, не теряя общности, тогда как вторая играет роль параметра в получающейся системе. В примерах

$$
\begin{gathered}
\left(u_{\tau_{i}}-u_{\tau_{j}}\right) u_{\tau_{i} \tau_{j}}+\left(u_{\tau_{j}}-u_{x}\right) u_{x \tau_{j}}+\left(u_{x}-u_{\tau_{i}}\right) u_{x \tau_{i}}=0, \\
u_{\tau_{i} \tau_{j}}=\frac{u_{x \tau_{i}}-u_{x \tau_{j}}}{e^{u_{\tau_{i}}}-e^{u_{\tau_{j}}}}
\end{gathered}
$$

обе постоянных интегрирования, по-видимому, несущественны. 
Благодарности. Работа выполнена при поддержке РФФИ (гранты № 04-0100403, 06-01-92051-KЭ_а).

\section{Список литературы}

[1] L. Martínez Alonso, A. B. Shabat, Phys. Lett. A, 300:1 (2002), 58-64; J. Nonlinear Math. Phys., 10:2 (2003), 229-242; Л. Мартинес Алонсо, А.Б. Шабат, ТМФ, 140:2 (2004), 216-229; A. B. Shabat, L. Martínez Alonso, "On the prolongation of a hierarchy of hydrodynamic chains", New Trends in Integrability and Partial Solvability (Cadiz, Spain, 2002), NATO Sci. Ser. II. Math. Phys. Chem., 132, eds. A. B. Shabat et al., Kluwer, Dordrecht, 2004, 263-280.

[2] Е.В. Ферапонтов, К.Р. Хуснутдинова, М.В. Павлов, ТМФ, 144:1 (2005), 35-43; E. V. Ferapontov, K. R. Khusnutdinova, S. P. Tsarev, Commun. Math. Phys., 261:1 (2006), 225-243.

[3] E. V. Ferapontov, Phys. Lett. A, 158:3-4 (1991), 112-118.

[4] A. B. Shabat, J. Nonlinear Math. Phys., 12: Suppl. 1 (2005), 614-624.

[5] Н. Х. Ибрагимов, А. Б. Шабат, ДАН СССР, 244:1 (1979), 57-61.

[6] В.Е. Захаров, С.В. Манаков, С.П. Новиков, Л.П. Питаевский, Теория солитонов. Метод обратной задачи, Наука, М., 1980.

[7] A. Hone, V.S. Novikov, C. Verhoeven, Inverse Problems, 22:6 (2006), 2001-2020. 